\title{
IT-Integration in Zeiten von Digitalisierung - (k)ein alter Hut?
}

\author{
Melanie Huber $\mathbb{D} \cdot$ Christopher Rentrop $\mathbb{D} \cdot$ Stephan Zimmermann
}

Eingegangen: 1. November 2019 / Angenommen: 4. Mai 2020 / Online publiziert: 26. Mai 2020

(C) Der/die Autor(en) 2020

Zusammenfassung Durch die zunehmende Vernetzung und den Anstieg von eingesetzter Hard- und Software hat sich die Komplexität der Unternehmensarchitektur von Unternehmen über die Jahre stetig erhöht. Das Aufkommen nutzerfreundlicher Informationstechnologie (IT)-Lösungen befähigt außerdem Fachbereiche, IT innovativ einzusetzen. Dies erhöht die Heterogenität und damit nochmals die Komplexität der Unternehmensarchitektur. Darüber hinaus treibt dieser IT-Einsatz die Digitalisierung in den Unternehmen maßgeblich voran. Dies wirft die Frage auf, ob Unternehmen überhaupt noch eine Relevanz in der Reduktion der Komplexität durch IT-Integration sehen oder ob dies vor dem Hintergrund der Digitalisierung schon ein alter Hut ist. Experteninterviews und eine qualitative Datenanalyse zeigen, dass IT-Integration und Digitalisierung keine disjunkten Phänomene sind, sondern sich gegenseitig beeinflussen. Die Ergebnisse betonen, wie unterschiedlich der Begriff aufgefasst werden kann und dass die einheitliche Nutzung damit essenziell ist. Darüber hinaus zeigen sie, dass Digitalisierung einerseits Treiber der IT-Integration ist, andererseits aber auch die Möglichkeiten zur Umsetzung verändert. Dabei ist die Integrationsentscheidung durch die Vielzahl an Vor- und Nachteile komplex. Fachbereichs-IT ist selten explizites Ziel von IT-Integrationsprojekten. Der Beitrag zeigt den wissenschaftlichen Forschungsbedarf in neuen technologischen Möglichkeiten zur IT-Integration und in der Balance von Flexibilität und IT-Integration in der Unternehmensarchitektur. Er beleuchtet, dass eine gemeinsame Sprache die Basis für IT-Integrationsprojekte ist und dass eine Kultur, in der Fachbereiche aktiv an ITIntegrationsentscheidungen teilhaben, das Ziel eines jeden Unternehmens sein soll-

\footnotetext{
M. Huber $\cdot$ C. Rentrop $(\bowtie)$

Konstanzer Institut für Prozesssteuerung, HTWG Konstanz, Konstanz, Deutschland

E-Mail: rentrop@htwg-konstanz.de

S. Zimmermann

HS Augsburg, Augsburg, Deutschland
} 
te. Insgesamt zeigen die Analysen, dass IT-Integration noch lange kein alter Hut, sondern, im Gegenteil, brandaktuell ist.

Schlüsselwörter IT-Integration · Unternehmensarchitektur · Digitalisierung · Digitale Transformation $\cdot$ Fachbereichs-IT $\cdot$ Experteninterview

\title{
IT Integration in Times of Digitalization-Old News or a Hot Topic?
}

\begin{abstract}
The complexity of enterprise architectures of companies has increased in the last years, due to the usage of more hard-, and software and their growing interconnectedness. The rising of user-friendly information technology (IT) enables business departments to use IT in an innovative way. This increases the heterogeneity of enterprise architectures and fuels the transformation that digitalization demands of organizations. Here, the question is raised, if organizations see any value in reducing the complexity using IT integration or if this is old news considering digitalization. Expert interviews and a qualitative data analysis show that IT integration and digitalization are no disjoint phenomena but are rather mutually dependent. The results show how different the experts interpret the term and that uniform use is therefore essential. Digitalization is on the one hand driver of IT integration, but on the other hand it also changes its implementation. The integration decision is complex due to various advantages and disadvantages. Business-managed IT is only rarely the target of IT integration projects. This paper contributes to the scientific discussion by showing that new implementation techniques as well as balancing the benefits and drawbacks of an IT integration should be the target of future research. Additionally, a mutual language is the foundation for IT integration projects and a culture, where business units are part of IT integration decisions should be the goal of every company. Overall, the analyses show that IT integration is not old news but, in contrary, a hot topic.
\end{abstract}

Keywords IT Integration · Enterprise Architecture - Digitalisation · Digital Transformation $\cdot$ Business-Managed IT $\cdot$ Expert Interview

\section{Einleitung}

Kaum ein Thema ist derzeit so präsent im öffentlichen Diskurs wie die Digitalisierung. Digitalisierung im ursprünglichen Sinne, also der Umwandlung von analogen Signalen in digitale, ist schon seit dem Aufkommen der ersten Computer ein zentraler Begriff (Legner et al. 2017). Bei der aktuellen Debatte stehen jedoch vor allen Dingen aktuell diskutierte Ansätze, wie beispielsweise Künstliche Intelligenz, und ihr Wert für Wirtschaft und Gesellschaft im Zentrum (BDA und BDI 2019). Dabei handelt es sich nicht um eine rein technische Diskussion, sondern eine sozio-technische. Dies bedeutet, dass nicht nur die Technologieauswahl und -implementierung betrachtet werden darf. Vielmehr ist eine zweite wichtige Dimension der Mensch und die Auswirkungen der Technologie auf seinen Alltag und seine Arbeitsweise. Auch verschiedene Branchenverbände diskutieren, wie sich die Veränderungen aus der 
aktuellen Digitalisierung auf die Unternehmen und ihre Geschäftszweige auswirken (BDA und BDI 2019). Die Prozesse, die durch die Digitalisierung aufkommenden Technologien zu implementieren und im unternehmerischen Kontext zu nutzen, werden als digitale Transformation bezeichnet. ${ }^{1}$ Unternehmen müssen sich dabei mit den Auswirkungen auf ihre Strukturen, Geschäftsstrategien und -modelle sowie Unternehmensarchitektur auseinandersetzen (Legner et al. 2017). Eine Unternehmensarchitektur beschreibt die Informationstechnologie (IT)-Elemente wie Applikationen, Daten, Infrastruktur und ihre Verbindungen untereinander. Außerdem baut sie eine Brücke zu den Geschäftselementen und ist dabei ein essenzielles Instrument, um die Geschäftsstrategie umzusetzen (Keller 2017). Unternehmensarchitekturen unterliegen dabei sowohl unternehmensinternen Entscheidungen als auch externen Entwicklungen, wie dem Aufkommen nutzerfreundlicher IT-Lösungen. Diese erleichtern es den Fachabteilungen mehr und mehr, die Unternehmensarchitektur zu gestalten, indem sie IT beschaffen, innovativ einsetzen und damit die Digitalisierung vorantreiben (Kopper et al. 2018). Dies verhindert jedoch die Transparenz über die Unternehmensarchitektur und erhöht ihre Komplexität durch redundante Systeme (Huber et al. 2017). IT-Integration, also die Zusammenführung von Daten, Funktionen, Prozessen, Methoden oder Programmen (Mertens 2012), ist ein bekanntes und lang diskutiertes Mittel zur Verringerung dieser Redundanz. Dadurch, dass Fachbereiche die Digitalisierung durch den selbstständigen und innovativen IT-Einsatz vorantreiben, stellt sich die Frage, ob das Thema aktuell überhaupt noch relevant oder schon ein alter Hut ist. Daher ist das Ziel dieses Beitrags, den aktuellen Umgang von Unternehmen mit IT-Integration und die Rolle der Digitalisierung zu beleuchten. Er soll damit aufzeigen, ob und wie Digitalisierung als Treiber der IT-Integration wirkt, welche Auswirkungen die Digitalisierung auf die Integrationsentscheidung hat und welche Rolle die IT der Fachbereiche in der IT-Integration spielt.

In der aktuellen Forschung wird IT-Integration durchaus als Basis für digitale Gestaltungsfelder, Smart Cities (Pittaway und Montazemi 2018), digitale Plattformen (Schermuly et al. 2019) oder für die datengetriebene Produktion (Kammler et al. 2019) betrachtet. Besonders der Finanzsektor (Schmidt et al. 2017), aber auch der Gesundheitssektor (Hufnagl et al. 2019) weisen laut Untersuchungen eine mangelnde Integration von IT-Systemen für die Digitalisierung auf. Die von den Fachbereichen selbst beschafften IT-Lösungen, sogenannte Schatten-IT, stellt ein weiteres Diskussionsfeld in der anwendungsnahen Wissenschaft zur Digitalisierung dar (Huber et al. 2018). Die beschriebenen Forschungsarbeiten konzentrieren sich dabei auf Teilaspekte der IT-Integration im Kontext der Digitalisierung und zeigen damit die Verbindung der beiden Themen auf. Im Gegensatz zu den genannten Artikeln fokussiert dieser Beitrag jedoch nicht auf einzelne Branchen oder Technologien, sondern liefert ein breiteres Bild. Damit leitet er Herausforderungen ab und stellt den Zusammenhang von IT-Integration und Digitalisierung generell dar. Die nachfolgenden Analysen weisen den Forschungsbedarf darin auf, als dass eine Balance zwischen Flexibilität und IT-Integration zu finden ist. Darüber hinaus

\footnotetext{
${ }^{1}$ Im allgemeinen Sprachgebrauch werden die Begriffe digitale Transformation und Digitalisierung für den Wandel der Unternehmen oft synonym verwendet, (Legner et al. 2017). In diesem Beitrag wird daher durchgehend das Wort Digitalisierung genutzt.
} 
verändern neue Technologien die Umsetzung von IT-Integration. Der Beitrag beschreibt, dass Unternehmen besonderen Wert auf eine gemeinsame Sprache, Kultur und Governance zu legen haben, um IT-Integration im Kontext von Digitalisierung erfolgreich umzusetzen.

Das folgende Kapitel erläutert, wie sich die Komplexität von Unternehmensarchitekturen durch interne und externe Einflüsse immer weiter erhöht hat. Es beschreibt, wie sich dadurch die Anforderungen an die Unternehmensarchitektur verändert haben und verdeutlicht an einem Praxisbeispiel, wie sich damit auch die Frage nach IT-Integration ändert. Der Schluss des Kapitels bildet die Formulierung von Forschungsfragen, die sich auf IT-Integration im Kontext der aktuellen Digitalisierung beziehen. Das dritte Kapitel beschreibt den Forschungsansatz, der sich aus leitfadengesteuerten Interviews und aus qualitativer Kodierung zusammensetzt. Im Anschluss folgt die Darstellung der Ergebnisse und die Diskussion dieser im Hinblick auf die gestellten Forschungsfragen. Der Beitrag endet mit einem abschließenden Fazit, Empfehlungen und Ausblick.

\section{Unternehmensarchitektur im Wandel}

Seit dem ersten Einsatz von IT in den Unternehmen verändert sich die Unternehmensarchitektur stetig (Laudon et al. 2010): Mitte des 20. Jahrhunderts bestand sie noch aus einzelnen Großrechnern und später aus dezentralen Minicomputern. In den 1970er Jahren verbreiteten sich die ersten Personal Computer (PC) in den Unternehmen durch Programme zur Text- und Datenverarbeitung. Ende der 1990er Jahre öffneten sich Unternehmensarchitekturen durch das Internet nach außen. Unternehmen konnten sich jetzt nicht nur mit ausgewählten Partnern vernetzen, sondern auch mit verschiedenen Kunden und Lieferanten durch Web-Standards und Electronic Data Interchange. Außerdem wurden auch die internen Unternehmensarchitekturen komplexer. Heutzutage nutzen Mitarbeiter PCs für komplexe Systeme, zum Beispiel in einer Client-Server-Architektur. Oft arbeiten sie außerdem mit mobilen Endgeräten wie Smartphones oder Tablets. Doch nicht nur die Anzahl und Komplexität von Hardwareelementen steigt, sondern auch die von Software. IT-Lösungen werden immer nutzerfreundlicher. Die sogenannte Consumerization erleichtert es den Mitarbeitern, den Wert von IT für ihre Tätigkeiten zu erkennen und sie einzusetzen (Niehaves et al. 2013). Dadurch beschaffen Fachbereiche IT selbst und gestalten auf diese Weise die Unternehmensarchitektur durch Einsatz von Schatten-IT erheblich mit (Haag und Eckhardt 2017). Das Management von Unternehmensarchitekturen beschäftigt sich eben mit der Unternehmensarchitektur als Ganzes, es behält einen Überblick über die eingesetzten Applikationen, ihre Schnittstellen und die darunterliegende IT-Infrastruktur über das gesamte Unternehmen hinweg. Es erhebt die Ist-Architektur, plant auf Grund von festgelegten Standards und Prinzipien, wie die Soll-Architektur aussehen soll und initiiert Projekte, um diese zu erreichen. Fachbereiche haben oft keinen Überblick über die Ist- und Soll-Architektur, wie sie von der zentralen Stelle definiert und geplant wurde. Daher passt die von ihnen selbst beschaffte oder entwickelte IT häufig nicht in die aktuelle oder geplante Unter- 
nehmensarchitektur, was zu Redundanzen und Inkonsistenzen führt (Huber et al. 2017).

Während Unternehmensarchitekturen immer komplexer werden, haben sich die Anforderungen an diese ebenfalls gewandelt. Die Diskussion um die Digitalisierung spiegelt diesen Wandel wider. Einerseits soll Innovation durch den wertbringenden Einsatz neuer Technologien in den Unternehmen vorangetrieben werden (Legner et al. 2017). Als Ergebnis bedeutet dies Freiheit in der Wahl der IT-Systeme und der Gestaltung der Prozesse. Erst mit dieser Freiheit können Mitarbeiter selbst gewählte neue Technologien einsetzen, evaluieren und selbstständig Prozesse verändern. Dies führt jedoch unmittelbar zu einer ansteigenden Heterogenität und Komplexität in der Unternehmensarchitektur (Huber et al. 2017). Andererseits erfordern andere Ziele der Digitalisierung jedoch ein hohes Maß an Automatisierung und damit eine hohe Homogenität und IT-Integration. So kann zum Beispiel ein Ziel eine starke Ausrichtung am Kunden sein, damit dieser auf verschiedenen Kanälen mit dem Unternehmen interagiert (Bender und Gronau 2017). Da diese Interaktion möglichst fehlerfrei und schnell ablaufen soll, müssen die verschiedenen Kanäle automatisierten Zugriff auf Kunden- und andere relevante Daten haben. Ein anderes Ziel kann die Entwicklung hin zu einem datengetriebenen Geschäftsmodell sein, in dessen Fokus nicht mehr physische Produkte stehen, sondern Daten und darauf basierende Services. Dazu sind Daten aus verschiedenen internen IT-Systemen, aber auch aus externen Quellen oder bereits ausgelieferten Produkten zu sammeln (Pflaum und Schulz 2018). Die Analyse dieser Daten ist die Voraussetzung für das Anbieten datengetriebener Services. Auch dieses Ziel setzt ein hohes Maß an IT-Integration zwischen den verschiedenen IT-Systemen voraus.

Die Herausforderungen können durch ein Praxisbeispiel konkretisiert werden: Ein Automobilzulieferer mit 250 Mitarbeitern fertigt in verschiedenen Werken Standardsowie Individualprodukte. Im Hauptwerk gibt es fünf verschiedene Fertigungslinien. Für jede der Linien ist eine Kapazitätsplanung zu erstellen. Die Teamleiter der Linien entwickelten für diese Aufgabe jeweils ihre eigenen Planungen. Je nach technischer Fähigkeit der Teams sind diese verschieden komplex und mit unterschiedlicher technischer Basis umgesetzt. Die Anforderungen an die Planungen und die damit verbundenen Zielfunktionen sind dabei ebenfalls unterschiedlich: In Linie 1 werden große Serien gefertigt werden, hier liegt der Fokus auf der Auslastung der Maschinen. In Linie 2 werden kleinere Serien gefertigt, wobei das Ziel die Auslastung des Personals ist. Die Daten, welche in die jeweiligen Planungen einfließen, sind jedoch die gleichen. Es werden Personal- und Maschinendaten benötigt, die von den Teamleitern manuell in das jeweilige System eingegeben werden. Hier zeigt sich eine Redundanz der benötigten Daten, aber auch der Grundfunktionalität der Systeme. Getrieben von der Digitalisierung setzt das Unternehmen zunehmend auf eine automatisierte Fertigung. Die Bestellungen und Maschinenkapazitäten wurden bereits automatisiert. Daher steht das Unternehmen vor mehreren Herausforderungen. Die Kapazitätsplanungen werden manuell mit Daten angereichert, was einer automatisierten Fertigung im Wege steht. Des Weiteren sind die verschiedenen Planungen redundant zueinander. Dies legt eine Integration nahe. Ansonsten müsste alle einzelnen Systeme angebunden werden, was die Komplexität erhöhen und wiederum die Automatisierung behindern würde. Dabei müssen aber die Bedürfnisse der 
verschiedenen Linien beachtet werden. Eine bloße Integration unter Missachtung der unterschiedlichen Ziele kann dazu führen, dass die Teamleiter ihre Linien nicht mehr optimal steuern können oder das neue System sogar ablehnen. Daher steht das Unternehmen vor der Herausforderung, einerseits die bestehenden Kapazitätsplanungen zu integrieren und andererseits die Anforderungen der einzelnen Linien nicht zu missachten.

Dieses Beispiel zeigt, dass Unternehmen auf der einen Seite ein gewisses Maß an Freiheit zulassen müssen, wenn sie auf individuelle Anforderungen eingehen, oder wenn sie innovative Technologien entwickeln und einsetzen möchten. Auf der anderen Seite müssen sie Systeme sinnvoll miteinander integrieren, wenn ihr Ziel beispielsweise ein datengetriebenes Geschäftsmodell, eine hohe Kundenorientierung, oder, wie im obigen Beispiel, eine hohe Automatisierung ist. Eine ITIntegrationsentscheidung stellt sich somit unabhängig von der Unternehmensgröße, sondern ist in hohem Maße von den Zielen abhängig, die das Unternehmen mit dem Vorantreiben der Digitalisierung verfolgt (Timm et al. 2015). Die Herausforderung der Digitalisierung im Management der Unternehmensarchitektur ist es, abhängig von diesen Zielen, die IT-Systeme im richtigen $\mathrm{Ma} \beta$ zu integrieren.

In der wissenschaftlichen Diskussion wird die explizite Verbindung von IT-Integration und Digitalisierung noch wenig diskutiert. Die Kombination der Begriffe dienten als Suchwörter für die wissenschaftlichen Datenbanken SpringerLink, der Association for Information Systems eLibrary (AISeL) und Sciencedirect. Diese wurden ausgewählt, weil sie die wichtigsten Bücher, Zeitschriften und Konferenzbeiträge der Forschungsbereiche Wirtschaftsinformatik und Information Systems im deutschen und englischen Sprachraum beinhalten. Die Suche im Titel oder Abstract liefert eine kleine Anzahl an Artikeln (siehe Tab. 1). Die gefundenen Beiträge beschäftigen sich oft mit einer spezifischen Branche, zum Beispiel dem Gesundheitssektor (Houta et al. 2018; Hufnagl et al. 2019) oder dem Finanzwesen (Schmidt et al. 2017, 2016; Weinrich et al. 2016). Hier ist die mangelnde Integration ein Hindernis der Digitalisierung. Im Kontext von „Smart“ ist insbesondere die Datenintegration eine wichtige Grundlage. Fallbeispiele sind beschrieben in den Bereichen Smart Cities (An et al. 2018; Pittaway und Montazemi 2018), Smart Hotels (Lai und Hung 2017) oder Smart Services in der Produktion (Dreyer et al. 2017; Kammler et al. 2019). Andere behandelte Themen sind Schatten-IT (Huber et al. 2018), Supply Chain Integration (Korpela et al. 2017) oder digitale Plattformen (Bender und Gronau 2017; Pittaway und Montazemi 2018; Schermuly et al. 2019). Die gefundenen Publikationen behandeln nur einzelne Themengebiete oder Branchen. Daher stellt

Tab. 1 Wissenschaftliche Beiträge zu Digitalisierung und IT-Integration

\begin{tabular}{lllllll}
\hline & SpringerLink & & AISeL & & Sciencedirect & \\
& Suchergebnisse & Relevant & Suchergebnisse & Relevant & Suchergebnisse & Relevant \\
\hline 2019 & 0 & 0 & 35 & 2 & 3 & 0 \\
2018 & 1 & 1 & 40 & 2 & 1 & 0 \\
2017 & 0 & 0 & 44 & 5 & 1 & 0 \\
2016 & 0 & 0 & 24 & 2 & 1 & 0 \\
Summe & 1 & 1 & 143 & 11 & 6 & 0 \\
\hline
\end{tabular}


sich die Frage, ob das Thema noch eine Relevanz in der Praxis hat oder ob der Nutzen durch den selbstständigen IT-Einsatz die dadurch entstehende Heterogenität überwiegt. Die Beantwortung der nachfolgenden gestellten Forschungsfragen (FF) helfen dabei, die Untersuchung zielführend gestalten.

Erstens zeigt die Literatur, dass das Verständnis des Begriffes IT-Integration unterschiedlich ist (Chowanetz et al. 2012). Daher zielt die erste Frage auf das Begriffsverständnis ab:

FF1 Wie wird der Begriff IT-Integration in den Unternehmen verstanden?

Zweitens soll dieser Beitrag Treiber der IT-Integration identifizieren. Dabei stellt sich auch die Frage, ob Digitalisierung eine Rolle als treibender Faktor spielt.

FF2 Welche Faktoren treiben aktuell und zukünftig die IT-Integration?

Unternehmen müssen bei der Entscheidung über IT-Integration die Vor- und die Nachteile betrachten. Die dritte Frage dient dazu, herauszufinden, welche Faktoren Unternehmen hier beachten. Ein Ziel ist es auch, zu erläutern, wie sich die Digitalisierung auf die Faktoren auswirkt.

FF3 Welche Vor- und Nachteile sehen Unternehmen in IT-Integration?

Neben dem Abwägen der Vor- und Nachteile müssen Unternehmen die Entscheidung auch tatsächlich durchführen. Ob und inwieweit Digitalisierung eine Auswirkung auf dieses Abwägen hat, soll die folgende Frage ebenfalls beleuchten:

FF4 Welche Methoden zur Entscheidung über IT-Integration existieren?

Als letztes ist die steigende Anzahl von IT der Fachbereiche, getrieben durch nutzerfreundliche IT, eine aktuelle Herausforderung. Die fünfte Frage dient daher dazu, mehr über den Umgang der Unternehmen damit zu erfahren:

FF5 Welche Rolle spielt die IT der Fachbereiche bei der IT-Integration?

\section{Forschungsansatz}

Die oben genannten Forschungsfragen richten sich auf den aktuellen Stand der Praxis über IT-Integration. Daher bot sich für ihre Beantwortung das Experteninterview an. Dieses fragt Fachwissen ab, das die Experten aus ihrer beruflichen Tätigkeit erworben haben und daher repräsentativ für ihre Berufsgruppe weitergeben können. Da die Fragen offen gestellt sind und einen explorativen Charakter haben, eigneten sich leitfadengesteuerte Interviews zur Erhebung und einer qualitative Methodik zur Auswertung (Meuser und Nagel 2009).

Die Kriterien zur Auswahl der Interviewpartner waren zunächst, dass diese entweder in der IT-Abteilung eines Unternehmens, bei einem IT-Dienstleister oder als Wissenschaftler im Bereich der (Wirtschafts-)Informatik arbeiteten. Im Rahmen dieser Tätigkeit sollten sie sich mit Integrationsfragestellungen befassen oder in der Vergangenheit schon einmal befasst haben. Die Berufserfahrung in diesem Bereich musste mehr als fünf Jahre betragen. Außerdem war das Ziel, unterschied- 
Tab. 2 Interviewpartner

\begin{tabular}{|c|c|c|c|}
\hline Abkürzung & Aktuelle Funktion & Aktueller Arbeitsplatz & $\begin{array}{l}\text { Erfahrung } \\
\text { [Jahre] }\end{array}$ \\
\hline $\mathrm{CIO} 1$ & $\begin{array}{l}\text { Chief Information Officer } \\
\text { (CIO) }\end{array}$ & $\begin{array}{l}\text { Stahl- und Metallhändler, } 8500 \text { Mitar- } \\
\text { beiter (MA) }\end{array}$ & 30 \\
\hline $\mathrm{CIO} 2$ & $\mathrm{CIO}$ & Mobilität, $30.000 \mathrm{MA}$ & 10 \\
\hline CFO1 & $\begin{array}{l}\text { Chief Financial Officer } \\
(\mathrm{CFO})\end{array}$ & Maschinen- und Anlagenbau, 4000 MA & 20 \\
\hline $\mathrm{CFO} 2$ & $\mathrm{CFO}$ & Verteidigung, 500 MA & 6 \\
\hline MESI & $\begin{array}{l}\text { Manufacturing Executive } \\
\text { System (MES) Integrator }\end{array}$ & $\begin{array}{l}\text { Consulting, Kunden aus Mittelstand und } \\
\text { Konzerne }\end{array}$ & 30 \\
\hline ERPI & $\begin{array}{l}\text { Enterprise Resource Planning } \\
\text { (ERP) Integrator }\end{array}$ & Consulting, Kunden aus Handel & 25 \\
\hline ITAR & IT-Architekt & Energie, $12.000 \mathrm{MA}$ & 10 \\
\hline ENAR & Unternehmensarchitekt & Halbleiter, $3000 \mathrm{MA}$ & 12 \\
\hline PFEA & Professor & $\begin{array}{l}\text { Hochschule, Professur für Softwaretech- } \\
\text { nik und Architekturmanagement }\end{array}$ & 30 \\
\hline
\end{tabular}

liche Branchen und Unternehmensgrößen auszuwählen, um ein möglichst breites Bild zu erhalten. Überdies repräsentieren die Interviewpartner verschiedenen Perspektiven auf das Thema. Einerseits handelte es sich bei den Befragten um Geschäftsführer von Softwareintegratoren, die eine externe Sicht auf die Integration bestimmter Systeme in den Unternehmen liefern. Andererseits waren die Experten unternehmensinterne Personen, die sich mit IT-Integration beschäftigen, wie CIOs, aber auch CFOs, in deren Verantwortung das Thema IT-Integration liegt. Als letztes waren die Interviewpartner Stakeholder aus dem Bereich der Unternehmensarchitektur mit ihrer Sichtweise aus der Betrachtung der IT-Landschaft. Insgesamt sagten neun angefragte Experten zu (Tab. 2). Acht Befragungen wurden telefonisch und eine persönlich durchgeführt. Die Interviews dauerten zwischen 45 und $60 \mathrm{~min}$. Die

Tab. 3 Interviewfragen und dazugehörige Forschungsfragen

\begin{tabular}{ll}
\hline Forschungsfrage & Interviewfrage \\
\hline FF1 & Was verstehen Sie unter dem Thema IT-Integration? \\
FF2 & Wie wichtig ist das Thema Integration aktuell in den Unternehmen? \\
& Wie glauben Sie wird sich die Relevanz von Integration in den nächsten Jahren \\
& verändern? \\
FF3 & Welche Vorteile versprechen sich die Unternehmen von IT, welche Nachteile wol- \\
& len sie beheben? \\
& Gibt es auch Vorteile, Systeme nicht zu integrieren? \\
FF4 & Wie bewerten Sie, welche Systeme Sie (mit anderen, mit dem ERP-System, mit \\
& Wem MES-System) integrieren? \\
& Mit wem wird die Entscheidung abgesprochen? \\
& Wann taucht Schatten-IT auf? \\
FF5 & Inwieweit wird sie in Integrationsprojekte mit einbezogen? \\
& Was sind Herausforderungen in Bezug zur Integration? \\
& Gibt es Besonderheiten bei der Integrationsentscheidung?
\end{tabular}


Beantwortung der oben gestellten Forschungsfragen erfolgte mittels zwölf Interviewfragen (Tab. 3) ${ }^{2}$.

Die Datenanalyse umfasst eine händische Transkription der Interviews und den Import in das Tool MAXQDA ${ }^{3}$. Dieses unterstützt eine qualitative Kodierung (Corbin und Strauss 2014) und erlaubt, den Daten in mehreren Iterationen Codes zuzuordnen. Die Interviewfragen dienten dabei als grober Bezugsrahmen und somit als zu Beginn schon feststehende Codes. Die restlichen Codes entwickelten sich durch eine offene iterative Kodierung. Dabei wurden diese und die Auswahl der Codes in mehreren Iterationen von einem Forscher durchgeführt. Dies erfolgte so lange, bis die finalen Codes feststanden. Diese Codes wurden im Anschluss von den anderen Forschern einem Review unterzogen und weiter angepasst.

\section{IT-Integration als aktuelle und zukünftige Herausforderung}

Die nachfolgenden Abschnitte stellen die Ergebnisse der eben beschriebenen Analyse vor. Beginnend mit dem Begriff der IT-Integration, folgen danach die Treiber der Integration aktuell und in Zukunft. Der dritte Abschnitt stellt die geschilderten Vor- und Nachteile von IT-Integration dar. Danach folgt die Integrationsentscheidung und als fünftes die Ergebnisse der Fragen nach Fachbereichs-IT. Der letzte Abschnitt diskutiert die erläuterten Ergebnisse in Hinblick auf die fünf gestellten Forschungsfragen.

\subsection{Der Begriff der IT-Integration}

Einige der Interviewpartner bezeichneten den Begriff Integration in Bezug zu IT als divers und wiesen darauf hin, dass unterschiedliche Sichtweisen existieren. Trotzdem waren sich die Gesprächspartner einig, dass IT-Integration eine Zusammenführung darstelle und damit einen Austausch schaffe. PFEA: „Integration bedeutet die verschiedenen Teile in einer passenden Weise zusammenzuführen und miteinander in Interaktion zu setzen. " Ansonsten nannten die Interviewpartner unterschiedliche Aspekte der IT-Integration. So war eine genannte Dimension die Reichweite. Unternehmen könnten demnach entweder prozessübergreifend, über die IT-Landschaft oder über Organisationsgrenzen hinweg integrieren. Eine weitere Dimension ist die Richtung der IT-Integration, die vertikal oder horizontal sei und entweder asynchron oder synchron erfolgen könne. Ein Interviewpartner nannte überdies Enterprise Application Integration als Methode und die Technologien Enterprise Service Bus, Web Services und Interfaces. Die meisten der Experten schilderten, dass eine Integration auf verschiedenen Ebenen ablaufen könne. Ein besonderes Augenmerk legten die Teilnehmer dabei auf die Integration von Prozessen, aber auch von Daten und Systemen.

\footnotetext{
2 Die Experten erhielten für ihr Interview keine Entlohnung.

3 https://www.maxqda.de/.
} 


\subsection{Treiber und Bedeutung von IT-Integration heute und in Zukunft}

Die Interviewfragen nach den Treibern der IT-Integration bezogen sich zum einen auf aktuelle und zukünftige Treiber, zum anderen auf die Wichtigkeit des Themas aktuell und in Zukunft.

Als aktuellen Treiber nannten vier der Befragten explizit die Digitalisierung. Des Weiteren sind strategische Initiativen aktuell Anlass für IT-Integrationsprojekte. Weitere Faktoren sind die Reduktion von Kosten, Risiko oder Komplexität durch das Eliminieren ungewollter Redundanzen. Des Weiteren führten neue Geschäftsmodelle generell zu einem steigenden Integrationsbedürfnis. Die Experten schilderten, dass neue Technologien ausschlaggebend für ein steigendes Bedürfnis nach ITIntegration seien. Explizit nannten sie Trends wie Industrie 4.0, cyber-physische Systeme als Basis für die sogenannte vierte industrielle Revolution, und Internet of Things (IoT), also die Vernetzung von Gegenständen mit Hilfe des Internets, mit dem Ziel, dass diese kommunizieren und selbstständig Entscheidungen beispielsweise im Produktionsprozess treffen (Steinhoff 2016). Ein Interviewpartner sprach die Verschmelzung zwischen der IT- und der Operational Technology (OT), also der Hard- und Software zur direkten Überwachung und Steuerung der Leistung von Industrieanlagen oder Prozessen (Gartner 2020), an. ITAR: „Durch neue Digitalisierungsansätze, Trends wir Internet of Things, wachsen die beiden Welten immer stärker zusammen. Man spricht von einem IT-OT-Conversion-Effekt. Hier wird mehr Integration nötig werden." Die meisten Experten sagten, dass die Digitalisierung auch in Zukunft als Treiber eine große Rolle spiele. CFO2: „Digitalisierung treibt alle in die Systeme. "Ein Teilnehmer schilderte, wie sich ändernde Technologien wie Künstliche Intelligenz die IT-Integration verändern könnten. ITAR: „Ich denke, dass ein Thema wie Artificial Intelligence oder Machine Learning nicht nur aus EnduserSicht Relevanz bekommen wird in den nächsten Jahren. Auch die nicht sichtbaren Komponenten, wie Middleware, werden davon profitieren. “.

Alle Befragungsteilnehmer maßen dem Thema aktuell eine hohe Wichtigkeit bei. Auch für die Zukunft waren sich fast alle einig, dass das Thema die gleiche oder sogar eine höhere Wichtigkeit haben würde. CIO2: „Die Tendenz ist steigend. Wir bewegen uns in einer Welt, die zunehmend digitalisiert wird. Daher sprechen wir sehr intensiv darüber, zunehmend Prozesse zu digitalisieren. Was aber viel wichtiger ist als die Digitalisierung selbst, ist die vorgelagerte Prozessoptimierung. Ich glaube, dass das Thema zunehmend an Wichtigkeit gewinnt. Wer das heute noch nicht verstanden hat, der wird vielleicht in wenigen Jahren gar nicht mehr am Markt existieren."

\subsection{Vor- und Nachteile von IT-Integration}

Zur Strukturierung der Vor- und Nachteile einer IT-Integration erfolgt nachfolgend eine Unterteilung in die Ebenen Unternehmensstrategie, Unternehmensarchitektur, Prozessen, Systeme und Nutzer (Tab. 4).

Auf der strategischen Ebene nannten die meisten der Befragten den Vorteil der Kostensenkung durch Automatisierung. Diese liege vor allen Dingen in der Vermeidung von Doppeleingaben oder menschlichem Eingreifen. Ein weiterer Vorteil sei 
Tab. 4 Übersicht über Vorteile und Nachteile einer IT-Integration auf verschiedenen Ebenen

\begin{tabular}{lll}
\hline Ebene & Vorteile & Nachteile \\
\hline $\begin{array}{l}\text { Unternehmens- } \\
\text { strategie }\end{array}$ & $\begin{array}{l}\text { Kostensenkung durch Automatisierung } \\
\text { Bessere Kontrolle über aktuelle und zukünftige } \\
\text { Situation durch integriertes System } \\
\text { Einhaltung gesetzlicher Regularien }\end{array}$ & $\begin{array}{l}\text { Einmalige Kosten durch Integra- } \\
\text { tionsprojekt } \\
\text { Laufende Kosten durch Betrieb } \\
\text { des integrierten Systems }\end{array}$ \\
architektur & Einheitlichkeit von Prozessen und Daten & $\begin{array}{l}\text { Verringerung von Flexibilität } \\
\text { durch Verlust von Autonomie }\end{array}$ \\
& $\begin{array}{l}\text { Reduktion von Komplexität in der Unterneh- } \\
\text { mensarchitektur }\end{array}$ & $\begin{array}{l}\text { in der Gestaltung der IT-Land- } \\
\text { schaft }\end{array}$ \\
& Schaffung von Transparenz & \\
Prozesse & Wiederverwendbarkeit durch Modularität & Risiko bei Kopplung von Unter- \\
& Erhöhung der Effizienz von Prozessen & nehmensbereichen \\
Verbesserung von Prozessqualität & Weiterleitung von Fehlern \\
Systeme & Verbesserung von Kommunikation & Abhängigkeit von einem inte- \\
& Vermeidung von Systembrüchen & grierten System
\end{tabular}

die Kontrolle über die aktuelle, aber auch die zukünftige Situation. CFO1: „Im Nachgang von Mergers and Acquisitions (M\&A)-Aktivitäten ist es wichtig, die Kontrolle zu übernehmen. Das heißt, man muss rückwärts betrachtet wissen, was passiert ist. Im nächsten Schritt muss man Control übernehmen. Hier muss man eine Vorwärtsbetrachtung vornehmen, um zu lernen, Prozesse im Unternehmen zu steuern. "Integration helfe auch, gesetzliche Regularien einzuhalten. Demgegenüber stehen die Kosten, die durch eine IT-Integration entstehen. Diese setzen sich zusammen aus den Änderungen, die die Unternehmen an den Systemen vornehmen müssen und den zu erwarteten laufenden Kosten, die danach durch den Betrieb der integrierten Systeme entstehen.

Ein Vorteil einer IT-Integration auf der Ebene der Unternehmensarchitektur ist die Einheitlichkeit von Prozessen und Daten und die Reduktion von Komplexität. Dies erreichen Unternehmen durch die Eliminierung ungewollter Redundanzen und Standardisierung. Zudem schaffe IT-Integration Transparenz dadurch, dass Daten über Anwendungen hinaus sichtbar seien. ITAR nannte überdies den Vorteil der Wiederverwendbarkeit, die durch eine Modularität und der dadurch geschaffenen Flexibilität einzelner Elemente der Unternehmensarchitektur erreicht werde. Dagegen können Unternehmen aber durch IT-Integration auch eine gewisse Flexibilität und damit Schnelligkeit einbüßen. Ein Interviewpartner nannte dabei das Beispiel von Microservices. PFEA: „Mit modernen Architekturen wie Microservices nehmen Unternehmen Redundanzen und Nicht-Integration bewusst in Kauf, um autonome Einheiten zu schaffen, die für sich funktionieren können “. Darüber hinaus verliere das Unternehmen durch IT-Integration Autonomie in der Gestaltung der Architekturlandschaft.

Integration habe Vorteile für die Effizienz der Prozesse, wie die Verschlankung und Automatisierung. Außerdem würden Prozessqualität und Zusammenarbeit verbessert. Als Nachteil nannten die Befragten das Risiko, weil verschiedene Unterneh- 
mensbereiche absichtlich voneinander getrennt sind. ENAR: „Durch Middleware Bereiche zu koppeln, die unterschiedliche Risikobewertungen haben, ist nicht vernünftig. Hier sollte man separieren. “ Des Weiteren gehe mit einer Integration auch das Risiko einher, dass Fehler bei der Dateneingabe bei hoch integrierten Systemen weitergeleitet werden.

Vorteile für die Systeme ergeben sich durch die Vermeidung von Systembrüchen und die Ablösung ungeeigneter Systeme. Die Systemqualität steige auch dadurch, dass sich die Verfügbarkeit, die Schnelligkeit und die Zuverlässigkeit der Systeme verbessert. Integration senke die Risiken durch die Eliminierung von Spezialwissen und die Tatsache, dass mehr Nutzer ein System bedienen könnten. CFO1: „Mit einem Systemwechsel vermehrt sich die Anzahl der Personen, die die sich mit dem System auskennen. " Ein Nachteil auf der Ebene der Systeme sei die Abhängigkeit von einem einzelnen integrierten System.

Für den Nutzer kann eine IT-Integration eine verringerte Nachvollziehbarkeit bedeuten. Diese leidet bei einem hoch integrierten System, da sie wie eine Black Box agiere. Die Transparenz wird gesteigert, was für den Nutzer aber auch zum Problem werden kann. Außerdem gehe eine Individualität verloren. CIO2: „Bei Applikationen, die sehr individuell auf einen Fachbereich zugeschnitten sind, geht durch eine IT-Integration im Regelfall diese Individualität ein Stück weit verloren. “

\subsection{Methoden zur Entscheidung über IT-Integration}

Die Integrationsentscheidung ist eine individuelle Entscheidung für Unternehmen. Die meisten Befragten gaben an, einen Business Case zu bilden, der die Vor- und Nachteile gegeneinander aufwiegt. Daneben gilt es aber, noch die Entscheidung selbst zu beachten und die Art und Weise, wie die Bewertung vorgenommen wird.

Die Integrationsentscheidung erfolgt insbesondere bei den Gesprächspartnern aus dem Bereich der Unternehmensarchitektur und der Systemintegratoren systematisch, in anderen Fällen eher pragmatisch. Weiterhin zeigten sich zwei Vorgehensweisen. Eine Gruppe gab an, die Integrationsentscheidung vom System her zu treffen. Sie schilderten, dass das integriert würde, was im Funktionsumfang liege oder Kernkompetenz sei. ERPI: „Das ergibt sich aus dem ERP System. Der Leistungsumfang des Systems gehört in ein ERP-System. “ Die Ähnlichkeit und Redundanz zu vorhandenen Systemen spielten hier eine Rolle. Im Gegensatz zur ersten Gruppe betonten die beiden CIOs die Prozesse. CIO2: „Unsere Aufgabe ist es, durch intelligente Lösungen Geschäftsprozesse bestmöglich zu unterstützen. Daher muss der Prozess bei der Entscheidung im Mittelpunkt stehen. “

Um die Bewertung vorzunehmen, gaben die Teilnehmer die Möglichkeiten an, einen Fragebogen zu versenden oder Workshops abzuhalten. Die Identifikation der $\mathrm{zu}$ integrierenden Systeme erfolge entweder durch eine systematische Suche oder eine aktive Benachrichtigung durch die Fachverantwortlichen. Beteiligte bei der Entscheidung seien die Leitungsebene, also IT-Leitung und Manager. Des Weiteren aber auch operative Mitarbeiter wie Entwickler oder Process Owner. Die Hälfte der Experten sagten aus, dass Fachabteilungen bei der Entscheidung aktiv mitwirkten. Probleme bei der Bewertung ergeben sich vor allem aus der Frage, wer die Bewertung vornimmt. PFEA: „Wenn Sie es selbst bewerten, machen Sie sich angreifbar. 
Wenn Sie sich die Bewertung zuliefern lassen, müssen Sie sehr sorgfältig sein, wie Sie die Kriterien definieren. Sie müssen ein sehr klares Bewertungsraster vorgeben, damit kein Interpretationsspielraum herrscht. " Darüber hinaus nannten die Interviewten Machtaspekte, die eine Rolle spielten.

\subsection{Integration der IT der Fachbereiche}

In diesem Abschnitt geht es darum, wann die Frage nach der Integration von IT aus dem Fachbereich auftaucht und welche spezifischen Herausforderungen bei ihrer Integration zu beachten sind.

Die Frage nach Integration von IT der Fachbereiche stellt sich bei der Mehrheit der Interviewpartner im Zuge anderer Projekte. Dies geschehe entweder bei der Voranalyse oder während des Projekts selbst. ,Schatten-IT kann ich zu Beginn eines Projektes nicht auf dem Radar haben. Das bedeutet, dass diese im weiteren Projektverlauf identifiziert werden muss. " Ein Experte schilderte, dass die Fachbereiche mit der Forderung nach einer Integration der Systeme auf ihn zu kämen. CFO2: „Am Anfang war es wichtig, die Leute abzuholen und ihnen die Notwendigkeit klar zu machen. Jetzt sind wir soweit, dass die Leute selbst merken, was notwendig ist. “

Die Interviewten sahen Herausforderungen bei der Integration von Schatten-IT. Bei der Frage nach den Verantwortlichkeiten stelle sich die Frage, ob die Systeme in der Hoheit der IT-Abteilung oder des Fachbereichs liegen sollten. Außerdem bestehe eine Schwierigkeit in fehlenden oder unklaren Ansprechpartnern. Eine weitere Herausforderung sei die Kultur. CIO2 nannte zudem die Balance zwischen Individualität und Standardisierung und den Widerstand in den Fachbereichen. SchattenIT könne das Projektziel von Integrationsprojekten in Gefahr bringen. Viele Experten sagten auch, dass eine Abbildung der Schatten-IT in der IT-Landschaft oft nicht möglich sei. Bei der Integrationsentscheidung selbst sahen die Hälfte der Befragten Unterschiede zu den Systemen der IT-Abteilung. Es sei schwieriger, den Nutzen zu rechnen und die Qualität der Systeme sei geringer, wie MESI beschreibt: „,Wenn eine Lösung Schatten-IT ist, dann ist es eher ein Ablösekandidat. Es existiert kein vernünftiger Support, es gibt keine vernünftigen Strukturen, es ist nicht eingebunden in die IT-Infrastruktur. “

Die Hälfte der Befragten sagte, dass IT der Fachbereiche indirekt oder gezielt in Integrationsprojekte mit einbezogen werden sollte. Unterschiede zu Systemen der IT-Abteilung waren zu erkennen. Zwei Experten erwähnten, dass das Maß nicht verloren gehen dürfe und nicht alles eliminiert werden könne. Ein wichtiger Punkt, der von der Hälfte der Teilnehmer genannt wurde, ist die Rolle der Kultur. Gerade bei Schatten-IT solle das Management auf Kollaboration und Offenheit setzen. CFO2: „Sie müssen ihren Mitarbeitern Vertrauen geben, ihnen zuhören, aber auch um Verständnis werben, wenn eine Zeit lang kein Geld für Investitionen da ist. Damit können Sie sehr viel erreichen. "Wichtige Unterschiede zu den oben genannten Integrationskriterien sind die Wahrung der Flexibilität. Es brauche eine Balance zwischen Freiraum und einem gewissen Maß an Richtlinien. PFEA: „Ich erlaube der Fachseite ein gewisses Maß an Freiraum, handele mit ihr aber auch rote Linien aus und mache ihnen Angebote, damit sie auch ohne große Anstrengung innerhalb der roten Linien bleiben kann. “ 


\subsection{Diskussion der Ergebnisse}

Dieser Abschnitt fasst die vorherigen Aspekte zusammen. Tab. 5 zeigt die Ergebnisse in der Übersicht.

Die in den letzten Abschnitten genannten Aussagen beantworten die erste Forschungsfrage: „Wie wird der Begriff IT-Integration in den Unternehmen verstanden?". Sie zeigen, dass Integration vor allem als Schaffen einer Verbindung und der Gewährleistung eines Austausches zu verstehen ist. Jedoch gibt es Unterschiede bei den Integrationsobjekten. Die Interviewteilnehmer nannten am häufigsten Daten, Funktionen oder Prozesse als Ziel einer IT-Integration. Diese Objekte decken sich mit existierenden Schemata aus der Literatur, wobei hier zusätzlich noch Methoden und Programme auftauchen (Mertens 2012). Das diverse Verständnis des Integrationsobjekts weist dabei auf die unterschiedlichen Stakeholder hin, auf die sich eine IT-Integration auswirkt. So sind bei Integrationsprojekten auf organisatorischer Ebene die Fachbereiche wichtig. Bei Projekten auf einer technischen Ebene kann ihre Einbeziehung weniger relevant sein. Eine Analyse der Stakeholder und dementsprechende Beteiligung sind daher notwendig, wie auch die Forschung zu Digitalisierungsprojekten zeigt (Schmidt et al. 2017). Als Grundlage müssen Unternehmen eine gemeinsame Sprache schaffen. Damit einher gehen eine klare Definition und einheitliche Nutzung des Begriffs IT-Integration als die technische und/ oder organisatorische Zusammenführung von Daten, Funktionen, Prozessen, Methoden, Programmen oder einer Kombination dieser zur Schaffung eines Austauschs. Passend für den jeweiligen Anwendungsfall müssen Unternehmen die Definition konkretisieren, kommunizieren und konsistent nutzen.

Die zweite Frage lautete , Welche Faktoren treiben aktuell und zukünftig die IT-Integration?“. Als Antwort ergibt sich aus dem Input der Befragungsteilnehmer, dass die Treiber als unternehmensindividuell beschrieben sind. Trotzdem gibt es einige oft genannte Faktoren, wie die Reduktion von Kosten, Komplexität oder Risiko. Die Allgemeingültigkeit dieser Faktoren lässt darauf schließen, dass das Thema auch

Tab. 5 Zusammenfassende Darstellung der Ergebnisse

\begin{tabular}{ll}
\hline Forschungsfrage & Zusammenfassende Antwort \\
\hline $\begin{array}{l}\text { FF1: Wie wird der Begriff IT-Integration in } \\
\text { den Unternehmen verstanden? }\end{array}$ & $\begin{array}{l}\text { Schaffen einer Verbindung } \\
\text { Gewährleistung eines Austausches } \\
\text { Integrationsobjekte: Daten, Funktionen, Prozesse }\end{array}$ \\
$\begin{array}{l}\text { FF2: Welche Faktoren treiben aktuell und } \\
\text { zukünftig die IT-Integration? }\end{array}$ & Unternehmensindividuell \\
& Übergreifende Faktoren: Reduktion von Kosten, Kom- \\
& plexität und Risiko, Digitalisierung \\
FF3: Welche Vor- und Nachteile sehen & Vor- und Nachteile auf Ebene Strategie, Unterneh- \\
Unternehmen in IT-Integration? & mensarchitektur, Prozesse, Systeme und Nutzer \\
& Übergreifende Vorteile: Kostensenkung, Erhöhung von \\
& Flexibilität, Systemqualität \\
& Übergreifende Nachteile: Implementierungskosten, \\
& Senkung von Flexibilität, Verlust von Individualität \\
FF4: Welche Methoden zur Entscheidung & Abwägen von Kosten und Nutzen \\
über IT-Integration existieren? & Vorgehensweise: pragmatisch oder systematisch \\
FF5: Welche Rolle spielt IT der Fachberei- & Eher Nebenprodukt, selten explizit Teil des Projekts \\
che bei IT-Integration? & Hauptprobleme: Governance, Kultur, Aufwand \\
\hline
\end{tabular}


in Zukunft relevant bleiben wird. Digitalisierung spielt dabei sowohl aktuell als auch in Zukunft eine große Rolle, weil mangelnde IT-Integration ein Hindernis für die Digitalisierung in den Unternehmen ist, insbesondere im Gesundheits- (Hufnagl et al. 2019) und im Bankensektor (Schmidt et al. 2016). Dabei ist IT-Integration die Basis, um beispielsweise neue Geschäftsmodelle zu entwickeln, wie auch aktuell in der Forschung beschrieben (Bender und Gronau 2017). Genauso verändern aber auch neue Technologien die Art, wie Unternehmen IT-Integration besser und automatisierter umsetzen können.

Zur Beantwortung der Frage ,Welche Vor- und Nachteile sehen Unternehmen in IT-Integration? " lässt sich sagen, dass die Experten eine Vielzahl an Vor- als Nachteile beschrieben, wobei sie deutlich mehr Vorteile nannten. Die Vor- und Nachteile wirken sich auf alle Bereiche des Unternehmens aus. Ein übergreifender Faktor sind die Kosten. Diese bestehen aus den einmaligen, sowie den laufenden Kosten, wie auch in der IT-Integrationsliteratur erwähnt (Khoumbati et al. 2006). Eine erfolgreiche IT-Integration führt aber langfristig zu einer Senkung der Kosten, etwa durch eine höhere Automatisierung und Effizienz in Prozessen und Systemen. Zweischneidig verhält es sich mit dem genannten Faktor der Flexibilität. Einerseits kann ITIntegration zu einem Zugewinn führen. Andererseits kann sie aber auch Flexibilität in der IT-Landschaft behindern, was den Zielen von Digitalisierungsinitiativen entgegenwirkt (Fürstenau und Glaschke 2015). Die Qualität und Verfügbarkeit von Systemen können sich durch eine Integration erhöhen, jedoch kann der Verlust an Individualität zu emotionalen Widerständen bei den Nutzern führen. Hier gilt es, eine geeignete Technologie zu wählen und mit Change-Management den Ängsten zu begegnen (Schmidt et al. 2016).

Die Antworten zur vierten Frage, ,Welche Methoden zur Entscheidung über IT-Integration existieren? “ zeigen, dass das klassische Abwägen von Kosten und Nutzen auch bei der IT-Integration ein Mittel der Wahl ist. Die Vielzahl der zuvor genannten Vor- und Nachteile zeigen, dass es sich hier um ein komplexes Unterfangen handelt (Huber et al. 2018). Unternehmen müssen alle Faktoren kennen, sie messen und gegeneinander aufwiegen. Oft fehlt die Zeit, dies systematisch durchzuführen, so dass Unternehmen einen pragmatischen Ansatz wählen. Dies birgt die Gefahr, wichtige Faktoren und damit Interessen zu vergessen, womit wiederum die Akzeptanz sinken kann. Aufgrund der steigenden Anzahl von Elementen in der Unternehmensarchitektur sowie der wachsenden Heterogenität wird die Digitalisierung die Komplexität eines solchen Unterfangens noch erhöhen (Huber et al. 2017). Daher ist es wichtig, erfahrene Projektleiter zu wählen, die sicherstellen, dass die Ist-Situation ausreichend analysiert wird, darauf aufbauend die richtigen Entscheidungen getroffen und die richtigen Stakeholder einbezogen sind.

Die Ergebnisse beantworten die fünfte Forschungsfrage: ,Welche Rolle spielt IT der Fachbereiche bei IT-Integration? “. Erstens wird IT der Fachbereiche als Nebenprodukt in Integrationsprojekten betrachtet, ansonsten wird selten systematisch nach ihr gesucht. Dies könnte am mangelnden Bewusstsein über die Risiken und Prozessbedeutung von Schatten-IT in den Unternehmen liegen (Fürstenau et al. 2016). Als zweites wird klar, dass insbesondere das Problem oft in der Governance liegt. Dies könnten Unternehmen durch eine Identifikation und Bewertung der Systeme regeln (Zimmermann et al. 2017). Als drittes zeigen die Analysen, dass Unternehmen ein 
Bewusstsein für die Bedeutung von Kultur haben. Die richtige Kultur ist im Rahmen einer angestrebten Digitalisierung ein wichtiges Schlüsselelement (Eden et al. 2019). Eine aufgeschlossene Kultur, in der Mitarbeiter selbst Systeme zur Integration anregen, sollte das Ziel jedes Unternehmens sein. Als viertes ist es wichtig, dass Unternehmen bei der Integration von Schatten-IT den großen Zeitaufwand beachten. Eine Vorauswahl auf Grund von Kriterien wie Risiko, Größe oder das Clustern verschiedener Systeme ist hilfreich, um den Aufwand zu verringern.

Das zu Beginn eingeführte Beispiel des mittelständischen Automobilzulieferers mit der heterogenen Fachbereichs-IT-Landschaft kann nun aus den gerade beschriebenen Blickwinkeln betrachtet werden. So ist, sowohl bei der Analyse der Entscheidung als auch später bei der Projektdurchführung, eine eindeutige Benennung des Integrationsvorhabens wichtig. In der Analyse ist klar zu kommunizieren, dass eine einheitliche Lösung für die Kapazitätsplanung gefunden werden soll, welche die Personal- und Maschinendaten integriert und in die Planung mit einbezieht. Das Beispiel beschreibt, dass mehrere redundante Systeme mit gleicher oder einer ähnlichen Funktionalität bestehen. Somit ist ein Treiber die Reduktion dieser ungewollten Komplexität. Diese Komplexität bestand jedoch schon länger. Die Digitalisierung und der damit aufkommende Wunsch nach einer automatisierten Fertigung ist jedoch erst der konkrete Auslöser für die Suche nach einer integrierten Lösung. Hier zeigt sich die große Relevanz der Digitalisierung als Treiber von Integrationsprojekten.

Bei der Suche nach einer Lösung muss das Unternehmen die Vor- und Nachteile der Integration der Systeme betrachten. Vorteile wären zum Beispiel die Kostensenkung, die sich durch die automatisierte Planung und die gesparten Ressourcen der Teamleiter ergibt. Außerdem reduziert sich die Komplexität in der Architektur durch die Eliminierung der unterschiedlichen Systeme und es entsteht eine unternehmensweite Transparenz über die Planung. Die Wartbarkeit der Systeme erhöht sich, weil es sich nicht um mehrere individuell entwickelte Systeme handelt, sondern um ein vereinheitlichtes. Durch die Eliminierung der manuellen Dateneingabe erhöht sich die Effizienz der Prozesse. Nachteile wären zum Beispiel die Kosten, die durch eine Softwareeinführung und -anbindung oder eine Individualentwicklung entstehen würden, sowie die laufenden Betriebskosten des Systems durch die IT-Abteilung. Außerdem wird die Autonomie in der Planung der einzelnen Linien verringert. Die einzelnen Nutzer könnten einen Verlust an Individualität verspüren. Die Nachvollziehbarkeit der Berechnungen könnte sich verringern, was zu einem Widerstand gegen das System führen könnte. Obwohl das Ziel der automatisierten Fertigung die Integration der Systeme nahelegt, sollte das Unternehmen trotzdem die genannten Vor- und Nachteile im Blick haben und diese im Rahmen des weiteren Vorgehens bei der Auswahl der Technologie beachten. Eine Befragung der Nutzer des aktuellen Systems sowie eine Analyse der Funktionalitäten der einzelnen Individuallösungen sind notwendig. Darauf aufbauend ist es wichtig, ein geeignetes System auszuwählen, das die Bedürfnisse der Produktion möglichst gut erfüllt sowie auch die Anforderungen an die Anbindung des neuen Systems im Blick hat. Das Projekt sollte Teamleiter und Disponenten von Anfang an mit einbeziehen, um einen reibungslosen Ablauf der Implementierung und der Nutzung des neuen Systems zu gewährleisten. 


\section{Fazit, Empfehlungen und Ausblick}

Dieser Beitrag beleuchtete die Rolle von IT-Integration in Zeiten von Digitalisierung in Unternehmen. Die Ergebnisse zeigen, dass Digitalisierung eine große Rolle als Treiber von IT-Integration spielt und auch in Zukunft spielen wird. Die wachsende Anzahl an Elementen einer Unternehmensarchitektur und damit der erhöhten Anzahl an Beteiligten stellt dabei eine große Herausforderung dar. Unternehmen müssen die verschiedenen Stakeholder in IT-Integrationsprojekte und Kommunikationsmaßnahmen zielgruppengerecht einbeziehen. Außerdem zeigen die Ergebnisse, dass für die IT-Integration die Flexibilität ein wichtiger Faktor ist. Diese wird durch IT-Integration verringert, was aber der höheren Standardisierung und Homogenität dient. Darüber hinaus ist Flexibilität aber insbesondere dann wichtig, wenn schnell auf Marktänderungen zu reagieren ist. Unternehmen müssen mit Hilfe von Technologieentscheidungen das richtige Maß an Flexibilität finden. Des Weiteren ist die Kultur in Unternehmen ein besonders wichtiger Faktor. IT der Fachbereiche ist in IT-Integrationsprojekte mit einbezogen werden. Dazu sollte die Kultur genutzt werden, damit Fachbereiche selbst mögliche Integrationskandidaten melden und eine enge Zusammenarbeit mit der IT-Abteilung herrscht.

Die Erkenntnisse tragen zur wissenschaftlichen Diskussion bei, da das Thema ITIntegration aktuell eine hohe Bedeutung hat und diese in den nächsten Jahren weiter zunehmen wird. Daher ist es wichtig, sich mit dem Einfluss der Digitalisierung auf eine IT-Integration auseinanderzusetzen. Die Forschung zum Management von Unternehmensarchitekturen sollte der Frage nach der Balance zwischen Standardisierung und Individualität nachgehen. Dabei wirkt der Zusammenhang zwischen diesen aber auch umgekehrt. Neue Unternehmensthemen wie Künstliche Intelligenz transformieren auch Integrationstechnologien, was ebenfalls in der Forschung betrachtet werden sollte.

Für Leser aus der Praxis liegen relevante Erkenntnisse in der Bedeutung der eher als weich bezeichneten Faktoren und in der Schaffung einer gemeinsamen Sprache, um mit den richtigen Stakeholdern zu kommunizieren und eine offene Kultur zu schaffen. IT-Abteilungen sollten mit Fachbereichen regelmäßig über Integrationspotenziale sprechen. Fachbereiche müssen auf IT-Abteilungen zugehen und ihnen Integrationskandidaten melden können. Dazu ist eine Sensibilisierung notwendig, für welche Systeme sich eine Integration lohnt. Sie sollten daneben eine Governance etablieren, welche die Verantwortung für die Systeme der Fachbereiche klar regelt. Dieser Beitrag zeigt auf, dass IT-Integration eine aktuelle und zukünftige Herausforderung in den Unternehmen darstellt und damit noch lange kein alter Hut ist.

Funding Open Access funding provided by Projekt DEAL.

Open Access Dieser Artikel wird unter der Creative Commons Namensnennung 4.0 International Lizenz veröffentlicht, welche die Nutzung, Vervielfältigung, Bearbeitung, Verbreitung und Wiedergabe in jeglichem Medium und Format erlaubt, sofern Sie den/die ursprünglichen Autor(en) und die Quelle ordnungsgemäß nennen, einen Link zur Creative Commons Lizenz beifügen und angeben, ob Änderungen vorgenommen wurden. 
Die in diesem Artikel enthaltenen Bilder und sonstiges Drittmaterial unterliegen ebenfalls der genannten Creative Commons Lizenz, sofern sich aus der Abbildungslegende nichts anderes ergibt. Sofern das betreffende Material nicht unter der genannten Creative Commons Lizenz steht und die betreffende Handlung nicht nach gesetzlichen Vorschriften erlaubt ist, ist für die oben aufgeführten Weiterverwendungen des Materials die Einwilligung des jeweiligen Rechteinhabers einzuholen.

Weitere Details zur Lizenz entnehmen Sie bitte der Lizenzinformation auf http://creativecommons.org/ licenses/by/4.0/deed.de.

\section{Literatur}

An X, Bai X, Chen H, Han W (2018) Effective big data integration in the development of smart cities in China: a digital continuity approach. In: 11th CONF-IRM Proceedings (Paper 8)

BDA, BDI (2019) 10 Ziele für ein starkes Europa. https://bdi.eu/media/publikationen/?topic= Digitalisierung\#/publikation/news/10-ziele-fuer-ein-starkes-europa/. Zugegriffen: 21. Jan. 2020

Bender B, Gronau N (2017) Coring on digital platforms - fundamentals and examples from the mobile device sector. In: 38th ICIS Proceedings (Paper 6)

Chowanetz M, Legner C, Thiesse F (2012) Integration: an omitted variable in information systems research. In: 20th ECIS Proceedings (Paper 227)

Corbin J, Strauss A (2014) Basics of qualitative research: techniques and procedures for developing grounded theory. SAGE, Thousand Oaks

Dreyer S, Olivotti D, Lebek B, Breitner M (2017) Towards a smart services enabling information architecture for installed base management in manufacturing. In: Wirtschaftsinformatik Proceedings, S 31-45

Eden R, Jones AB, Casey V, Draheim M (2019) Digital transformation requires workforce transformation. MIS Q Exec 18:4

Fürstenau D, Glaschke C (2015) Weighting of integration qualities in IS architectures: a production case. In: 23th ECIS Proceedings (Paper 53)

Fürstenau D, Rothe H, Sandner M, Anapliotis D (2016) Shadow IT, risk, and shifting power relations in organizations. In: 22nd AMCIS Proceedings, S 1-10

Gartner (2020) Operational technology (OT). https://www.gartner.com/en/information-technology/ glossary/operational-technology-ot. Zugegriffen: 28. Jan. 2020

Haag S, Eckhardt A (2017) Shadow IT. Bus Inf Syst Eng 59:469-473

Houta S, Rüsing L, Stein M, Wilking T (2018) Einsatz der eHealth-Plattform zur Optimierung des MDKManagements durch Digitalisierung und Integration von Systemen. In: Pfannstiel MA, Straub S (Hrsg) Dienstleistungscontrolling in Gesundheitseinrichtungen: Aktuelle Beiträge aus Wissenschaft und Praxis. Springer, Wiesbaden, S 303-319

Huber M, Zimmermann S, Rentrop C, Felden C (2017) The influence of shadow IT systems on enterprise architecture management concerns. In: 14th EMCIS Proceedings, S 461-477

Huber M, Zimmermann S, Rentrop C, Felden C (2018) Toward a conceptual decision framework for shadow IT integration. In: 24th AMCIS Proceedings, S 1-5

Hufnagl C, Doctor E, Behrens L, Buck C, Eymann T (2019) Digitisation along the patient pathway in hospitals. In: 27th ECIS Proceedings (Paper 193)

Kammler F, Hagen S, Brinker J, Thomas O (2019) Leveraging the value of data-driven service systems in manufacturing: a graph-based approach. In: 27th ECIS Proceedings (Paper 189)

Keller W (2017) IT-Unternehmensarchitektur. Dpunkt, Heidelberg

Khoumbati K, Themistocleous M, Irani Z (2006) Evaluating the adoption of enterprise application integration in health-care organizations. JMIS 22:69-108

Kopper A, Fürstenau D, Zimmermann S, Klotz S, Rentrop C, Rothe H, Strahringer S, Westner M (2018) Shadow IT and business-managed IT: a conceptual framework and empirical illustration. Int J IT Bus Alignment Gov 9:53-71

Korpela K, Hallikas J, Dahlberg T (2017) Digital supply chain transformation toward blockchain integration. In: 50th HICSS Proceedings

Lai W-C, Hung W-H (2017) Constructing the smart hotel architecture-A case study in Taiwan. In: ICEB 2017 Proceedings

Laudon KC, Laudon JP, Schoder D (2010) Wirtschaftsinformatik: Eine Einführung. Pearson, München 
Legner C, Eymann T, Hess T, Matt C, Böhmann T, Drews P, Mädche A, Urbach N, Ahlemann F (2017) Digitalization: opportunity and challenge for the business and information systems engineering community. Bus Inf Syst Eng 59:301-308

Mertens P (2012) Integrierte Informationsverarbeitung 1-Operative Systeme in der Industrie, 18. Aufl. Gabler, Wiesbaden

Meuser M, Nagel U (2009) Das Experteninterview - konzeptionelle Grundlagen und methodische Anlage. In: Pickel S, Pickel G, Lauth H-J, Jahn D (Hrsg) Methoden der vergleichenden Politik- und Sozialwissenschaft. Neue Entwicklungen und Anwendungen. VS, Wiesbaden, S 465-479

Niehaves B, Köffer S, Ortbach K (2013) IT consumerization under more difficult conditions. In: 14th Annual Int. Conf. on Digital Government Research, S 205-213

Pflaum A, Schulz E (2018) Auf dem Weg zum digitalen Geschäftsmodell. HMD 55:234-251. https://doi. org/10.1365/s40702-018-0401-2

Pittaway J, Montazemi AR (2018) Seeking the 'smart' in cities: managing the process of innovating with IT. In: AMCIS 2018 Proceedings

Schermuly L, Schreieck M, Wiesche M, Krcmar H (Hrsg) (2019) Developing an industrial IoT platform-trade-off between horizontal and vertical approaches

Schmidt J, Drews P, Schirmer I (2016) End-users' perspective on digitalization: a study on work order processing in the German banking industry. In: AMCIS 2016 Proceedings

Schmidt J, Drews P, Schirmer I (2017) Digitalization of the banking industry: a multiple stakeholder analysis on strategic alignment. In: AMCIS 2017 Proceedings

Steinhoff C (2016) Aktueller Begriff Industrie 4.0. Wissenschaftlicher Dienst. Deutscher Bundestag, Berlin

Timm F, Wißotzki M, Köpp C, Sandkuhl K (2015) Current state of enterprise architecture management in SME utilities. In: INFORMATIK 2015

Weinrich T, Muntermann J, Gregory RW (2016) Exploring principles for corporate digital infrastructure design in the financial services industry. In: PACIS 2016 Proceedings

Zimmermann S, Rentrop C, Felden C (2017) A multiple case study on the nature and management of shadow information technology. J Inf Syst 31:79-101 